LA-UR-11-11672

\title{
Physical Degrees of Freedom for Gauge Fields and the Issue of Spin
}

\author{
T. Goldman \\ Theoretical Division \\ Los Alamos National Laboratory \\ Los Alamos, NM 87545 USA
}

\begin{abstract}
The conflict between the physical degrees of freedom of gauge bosons and the Lorentz group irreps naturally used to describe their couplings to matter fields are illustrated and discussed, and applied to issues of linear and angular momentum.
\end{abstract}

PACS numbers: 11.15.-q, 14.70.Bh, 14.70.Dj 


\section{INTRODUCTION}

The measurement of the quark contribution to the spin of the proton by means of the axial vector current has led to concerns as the small result[1] appears to be in conflict with the quark picture of nucleon structure. However, the axial current is not identical to the spin contribution, and the naive quark model pictures do not include the relativistic effects that certainly occur for the light quarks. Furthermore, the issue has gotten entangled with the question of gauge invariant operators for angular momentum components. Here, I first remind the reader of the effect in hydrogenic atoms where the spin "loss" can be explicitly calculated for the electron contribution, then show the relativistic general result that, when viewed from a boosted frame, a fermion with a given spin orientation at rest appears to have lost some of that spin. Next, I show that there exists a spin-one irrep of the Lorentz group that carries only the physical degrees of freedom of a vector boson, and must have a ClebschGordan relation to the usual $(1 / 2,1 / 2)$ irrep used for gauge fields, thus implying that the physical and unphysical degrees of freedom of the latter can be unambiguously separated. I then turn to the issue of gauge invariant quantities and recall an old, but ignored problem for the eigenvalues of the Pauli Hamiltonian relative to that of Dirac. After this, I show how the usual separation of gauge invariant angular momentum components for QED is inconsistent since the components do not obey angular momentum commutation rules, but that the

problem is solved by using the decomposition of Ref.([2]) which depends on identifying and separating the physical and unphysical parts of the gauge field in its usual representation. The same separation applies to momentum operators in gauge theories, and I identify a physical momentum which is neither the canonical nor the mechanical momentum. This decomposition also solves the issue of the Pauli-Dirac Hamiltonian dichotomy. Finally, I apply this decomposition to the originating issue of angular momentum decomposition in QCD and briefly discuss the relation to other proposals, some of which are now very similar.

\section{SPIN, BOOSTS AND ANGULAR MOMENTUM}

Consider the wave function for the ground state solution to the Dirac equation for an electron bound to an "infinitely" heavy nucleus of charge $Z$ (as shown in any good text on 
quantum mechanics):

$$
\psi \propto\left[\begin{array}{c}
1 \\
0 \\
-\imath \frac{(1-\gamma)}{Z \alpha} \cos \theta \\
\imath \frac{(1-\gamma)}{Z \alpha} \sin \theta e^{\imath \phi}
\end{array}\right]
$$

where $\gamma=\sqrt{1-Z^{2} \alpha^{2}}$ and we ignore the radial wave function as irrelevant to our purpose here. The normalization of this wave function is determined by

$$
\psi^{\dagger} \psi \propto 1+\left[\frac{(1-\gamma)}{Z \alpha}\right]^{2}\left[(\cos \theta)^{2}+(\sin \theta)^{2}\right]
$$

so that whether we use $\Sigma_{3}=\gamma_{3} \gamma_{5}$ or the generator of rotations about the 3 -axis, $\sigma_{12}$, the matrix elements after angular integration is

$$
\frac{1}{1+\left[\frac{(1-\gamma)}{Z \alpha}\right]^{2}}<1
$$

The difference must be made up by the electron orbital angular momentum and the angular momentum of the virtual photons involved in the binding of the system, so that total angular momentum is conserved.

\section{A. Boosts}

Similarly, if we start with a spin up Dirac fermion in its rest frame, and then boost ourselves to a different frame, either in the spin direction or in the plane transverse to it, we observe components no longer corresponding to a spin up fermion:

$$
\psi=\left[\begin{array}{l}
1 \\
0 \\
0 \\
0
\end{array}\right] \rightarrow \sqrt{\frac{E+m}{2 m}}\left[\begin{array}{c}
1 \\
0 \\
0 \\
-\left(\frac{p}{E+m}\right)
\end{array}\right] \text { or } \rightarrow \sqrt{\frac{E+m}{2 m}}\left[\begin{array}{c}
1 \\
0 \\
\left(\frac{p}{E+m}\right) \\
0
\end{array}\right]
$$

where the first corresponds to boosting transversely and preserves the $\Sigma_{3}$, the axial current matrix element, but not the matrix element of $\sigma_{12}$, the generator of rotations, while the second case corresponds to boosting in the 3-direction, which preserves the matrix element of $\sigma_{12}$ but not $\Sigma_{3}$. 


\section{SPIN-ONE IRREPS OF THE LORENTZ GROUP}

A massive spin-one particle in the $(1,0)$ irrep of the Lorentz group with spin up has components[3]

$$
\psi \propto \frac{m}{\sqrt{2}}\left[\begin{array}{l}
1 \\
0 \\
0
\end{array}\right] \rightarrow \frac{m}{\sqrt{2}}\left[\begin{array}{c}
\frac{p^{+}}{m} \\
1 \\
\frac{m}{p^{+}}
\end{array}\right]
$$

where the arrow shows the effect of boosting along the 3-axis. Just as for Weyl spinors, a parity conserving form may be constructed by appending to the above spinor its Wigner conjugate in the $(0,1)$ irrep, produced by applying the matrix

$$
\left[\begin{array}{ccc}
0 & 0 & 1 \\
0 & -1 & 0 \\
1 & 0 & 0
\end{array}\right]
$$

so that in the infinite momentum or zero mass limit one obtains the spin-one analog of a massless Majorana spinor in the Wigner-Weyl representation. This has only two spin polarizations:

$$
\left[\begin{array}{c}
1 \\
0 \\
0 \\
0 \\
0 \\
1
\end{array}\right] \text { and }\left[\begin{array}{c}
0 \\
0 \\
1 \\
1 \\
0 \\
0
\end{array}\right] \text {, }
$$

both of which are transverse, and which are just what is needed for the physical degrees of freedom of a photon. The coupling to fermions must then have the structure

$$
\bar{\Psi} \Gamma^{\xi} \phi_{\xi} \Psi=\bar{\Psi} \gamma^{\mu} A_{\mu} \Psi
$$

where $\xi$ runs over 6 index values and I have written the rhs in terms of the conventional photon gauge field. There must exist a set of Clebsch-Gordan coefficients, $\tilde{\Gamma}_{\mu}^{\xi}$, that relate the two irreps so that the conventional photon field is given by

$$
A_{\mu} \equiv \tilde{\Gamma}_{\mu}^{\xi} \phi_{\xi}
$$

but now with only two independent degrees of freedom. The gauge redundancy is only available in the conventional photon $\left(\frac{1}{2}, \frac{1}{2}\right)$ irrep. 


\section{A. Aside on Gauge non-Invariance of the Pauli Hamiltonian}

Transformation of a Hamiltonian by a time-dependent unitary operator changes the spectrum[4]:

$$
\begin{aligned}
\imath \frac{\partial \psi^{\prime}}{\partial t} & =\left(U H U^{-1}-\imath U \frac{\partial U^{-1}}{\partial t}\right) \psi^{\prime} \\
& \equiv H^{\prime} \psi^{\prime} \quad \text { with } \\
\psi^{\prime}=U \psi ; U & =\exp [-\imath H f(t)] \quad \text { which produces } \\
<H^{\prime}> & =(1+\dot{f}) \Sigma_{n}\left|c_{n}\right|^{2} E_{n} \\
& \neq \Sigma_{n}\left|c_{n}\right|^{2} E_{n}=<H>
\end{aligned}
$$

However, this is exactly the transformation made by Ffoldy and Wouthuysen to transform from the Dirac to the Pauli Hamiltonian, with

$$
U=\exp [\beta \vec{\alpha} \cdot(\vec{p}-e \vec{A}) / 2 m]
$$

The problem is that

$$
\begin{aligned}
H_{P}= & \left(U H_{D} U^{-1}-\imath U \frac{\partial U^{-1}}{\partial t}\right) \\
\simeq & \beta\left[m+\frac{(\vec{p}-e \vec{A})^{2}}{2 m}\right]-e A_{0}-\frac{e}{2 m} \beta \vec{\sigma} \cdot \vec{B} \\
& -\frac{\imath e}{8 m^{2}} \vec{\sigma} \cdot \vec{\nabla} \times \vec{E}-\frac{e}{4 m^{2}} \vec{\sigma} \cdot \vec{E} \times \vec{p}-\frac{e}{2 m^{2}} \vec{\sigma} \cdot \vec{E}
\end{aligned}
$$

requires the last time derivative term to produce the gauge invariant

$$
\vec{E}=-\vec{\nabla} A_{0}-\frac{\partial \vec{A}}{\partial t}
$$

in the last line. That is, the result is only valid in Coulomb gauge where this last time derivative term of the vector potential term doesn't contribute because

$$
\vec{\nabla} \cdot \vec{A}=0 ; \vec{\sigma} \cdot \vec{\nabla} \times \frac{\partial \vec{A}}{\partial t}=0 ; \vec{\sigma} \cdot \frac{\partial \vec{A}}{\partial t} \times \vec{p}=0
$$

But the result must be valid in any gauge! Furthermore, if we chose this gauge, it would seem that the energies would not be Lorentz covariant. Fortunately, Manoukian[5] has shown that despite appearances, Coulomb gauge is actually Lorentz covariant! As for the gauge invariance, we will solve this problem along with the gauge invariant decomposition of orbital angular momentum in the next section. 


\section{GAUGE INVARIANT CANONICAL ANGULAR MOMENTUM}

The straightforward decomposition of angular momentum in QED is not gauge invariant:

$$
\begin{aligned}
\vec{J}_{Q E D} & =\vec{S}_{e}+\vec{L}_{e}+\vec{S}_{\gamma}+\vec{L}_{\gamma} \quad \text { where } \\
\vec{S}_{e} & =\int d^{3} x \psi^{\dagger} \frac{\vec{\Sigma}}{2} \psi ; \quad \vec{L}_{e}=\int d^{3} x \psi^{\dagger} \vec{x} \times \frac{1}{\imath} \vec{\nabla} \psi \\
\vec{S}_{\gamma} & =\int d^{3} x \vec{E} \times \vec{A} ; \vec{L}_{\gamma}=\int d^{3} x \vec{x} \times E^{i} \vec{\nabla} A^{i}
\end{aligned}
$$

but the components of the commonly described gauge-invariant form

$$
\begin{aligned}
\vec{J}_{Q E D} & =\vec{S}_{e}+\vec{L}_{e}^{\prime}+\vec{J}_{\gamma}^{\prime} \quad \text { where } \\
\vec{S}_{e} & =\int d^{3} x \psi^{\dagger} \frac{\vec{\Sigma}}{2} \psi ; \quad \vec{L}_{e}^{\prime}=\int d^{3} x \psi^{\dagger} \vec{x} \times \frac{1}{\imath} \vec{D} \psi \\
\vec{J}_{\gamma}^{\prime} & =\int d^{3} x \vec{x} \times(\vec{E} \times \vec{B})
\end{aligned}
$$

do not obey the canonical commutation relations for angular momentum, viz.

$$
\left[\left(\vec{x} \times \frac{1}{\imath} \vec{\nabla}\right)_{j},\left(\vec{x} \times \frac{1}{\imath} \vec{\nabla}\right)_{k}\right]=\imath \epsilon_{j k l}\left(\vec{x} \times \frac{1}{\imath} \vec{\nabla}\right)_{l}
$$

but

$$
\left[\left(\vec{x} \times \frac{1}{\imath}(\vec{\nabla}-\imath e \vec{A})\right)_{j},\left(\vec{x} \times \frac{1}{\imath}(\vec{\nabla}-\imath e \vec{A})\right)_{k}\right]=\imath \epsilon_{j k l}\left\{\left(\vec{x} \times \frac{1}{\imath}(\vec{\nabla}-\imath e \vec{A})\right)_{l}+e x_{l} \vec{x} \cdot(\vec{\nabla} \times \vec{A})\right\} .
$$

The extra term can be avoided if instead of the full gauge field, we define a part, $\vec{A}_{\text {pur }}$,

such that $\vec{\nabla} \times \vec{A}_{\text {pur }}=0$, which removes the last term. (See, e.g., Ref.([6]).) Thus, we have proposed the decomposition[2]

$$
\begin{aligned}
\vec{J}_{Q E D} & =\vec{S}_{e}+\vec{L}_{e}^{\prime \prime}+\vec{S}_{\gamma}^{\prime \prime}+\vec{L}_{\gamma}^{\prime \prime} \quad \text { where } \\
\vec{S}_{e} & =\int d^{3} x \psi^{\dagger} \frac{\vec{\Sigma}}{2} \psi ; \vec{L}_{e}^{\prime \prime}=\int d^{3} x \psi^{\dagger} \vec{x} \times \frac{1}{\imath} \vec{D}_{p u r} \psi \\
\vec{S}_{\gamma}^{\prime \prime} & =\int d^{3} x \vec{E} \times \vec{A}_{f y s} ; \quad \vec{L}_{\gamma}^{\prime \prime}=\int d^{3} x \vec{x} \times E^{i} \vec{\nabla} A_{f y s}^{i}
\end{aligned}
$$

which are all gauge invariant because $\vec{A}_{f y s}$ is, and where

$$
\begin{gathered}
\vec{A} \equiv \vec{A}_{f y s}+\vec{A}_{p u r}, \vec{D}_{p u r} \equiv \vec{\nabla}-\imath e \vec{A}_{p u r} \\
\vec{\nabla} \cdot \vec{A}_{f y s}=0, \vec{\nabla} \times \vec{A}_{p u r}=0
\end{gathered}
$$

Note, this is not the same as choosing Coulomb gauge; it separates out the physical (transverse) component of the photon field as we showed above must be possible since a pure 
spin-one field irrep exists in the Lorentz group. If the gauge field is presented in any fixed gauge, the physical part may be projected out by using the constraints in Eqs.(21) and the pure gauge part identified as $\vec{A}_{\text {pur }}=\vec{A}-\vec{A}_{f y s}$. The explicit construction is given by

$$
\begin{aligned}
\vec{A}_{f y s}(x) & =\vec{\nabla} \times \frac{1}{4 \pi} \int d^{3} y \frac{\vec{\nabla} \times \vec{A}(y)}{|\vec{x}-\vec{y}|} \\
A^{0}{ }_{\text {fys }} & =\int_{-\infty}^{x} d x_{i}\left(\partial^{i} A^{0}+\partial_{t} A^{i}-\partial_{t} A_{f y s}^{i}\right) \\
\phi(x) & =-\frac{1}{4 \pi} \int d^{3} y \frac{\vec{\nabla} \cdot \vec{A}(y)}{|\vec{x}-\vec{y}|}+\phi_{0}(x) \\
\vec{A}_{\text {pur }} & =-\vec{\nabla} \phi(x) ; A_{\text {pur }}^{0}=\partial_{t} \phi(x) ; \nabla^{2} \phi_{0}(x)=0
\end{aligned}
$$

\section{A. Aside on Linear Momentum}

In the same way as for angular momentum, the bypassed problem of linear momentum can be solved. Neither the canonical momentum, nor the mechanical momentum

$$
\vec{p}=m \dot{\vec{r}}+q \vec{A}=\frac{1}{\imath} \vec{\nabla} ; \vec{p}-q \vec{A}=m \dot{\vec{r}}=\frac{1}{\imath} \vec{D}
$$

satisfies both the commutation relation for linear momentum and gauge invariance. Since a gauge transformation only affects the time component and longitudinal part of the vector potential, the separation we identified above as pur and fys corresponds to "parallel" and transverse separation, hence we recognize the physical momentum as

$$
\vec{D}_{p u r}=\vec{p}-q \vec{A}_{\|}=\frac{1}{\imath} \vec{\nabla}-q \vec{A}_{\|}
$$

where $\vec{A}$ has been separated into parallel and perpendicular components in the same way as above

$$
\vec{\nabla} \cdot \vec{A}_{\perp}=0 ; \vec{\nabla} \times \vec{A}_{\|}=0
$$

This also completes the solution of the problem of the gauge invariant Hamiltonian for the hydrogen atom, where the physical Hamiltonian is given by

$$
H_{f y s}=H+q \partial_{t} \omega(x)=\frac{\left(\vec{p}-q \vec{\nabla} \omega-\vec{A}_{\perp}^{c}\right)^{2}}{2 m}+q \phi^{c}
$$

and $\omega(x)$ is the phase in the gauge change from the Coulomb values (labelled ${ }^{c}$ ) for the 4 -vector potential and $\vec{p}-q \vec{\nabla} \omega$ is identified as the physical momentum as above. 


\section{APPLICATION TO QCD}

Again, the natural decomposition of spin and orbital angular momentum is not gauge invariant [7], but the gauge invariant forms do not obey the canonical commutation relations for angular momenta. We have proposed

$$
\begin{aligned}
\vec{J}_{Q C D} & =\vec{S}_{q}+\vec{L}_{q}^{\prime \prime}+\vec{S}_{g}^{\prime \prime}+\vec{L}_{g}^{\prime \prime} \quad \text { where } \\
\vec{S}_{q} & =\int d^{3} x \psi^{\dagger} \frac{\vec{\Sigma}^{2}}{2} ; \quad \vec{L}_{q}^{\prime \prime}=\int d^{3} x \psi^{\dagger} \vec{x} \times \frac{1}{\imath} \vec{D}_{p u r} \psi \\
\vec{S}_{g}^{\prime \prime} & =\int d^{3} x \vec{E} \times \vec{A}_{f y s} ; \quad \vec{L}_{g}^{\prime \prime}=\int d^{3} x \vec{x} \times E^{i} \overrightarrow{\mathcal{D}}_{p u r} A_{f y s}^{i}
\end{aligned}
$$

where $\vec{A}$ is now a matrix quantity; the gauge covariant derivatives

$$
\vec{D}_{\text {pur }}=\vec{\nabla}-\imath g \vec{A}_{\text {pur }} ; \overrightarrow{\mathcal{D}}_{\text {pur }}=\vec{\nabla}-\imath g\left[\vec{A}_{\text {pur }}, \quad\right]
$$

and the defining constraints are

$$
\begin{array}{r}
\vec{D}_{p u r} \times \vec{A}_{p u r}=\vec{\nabla} \times \vec{A}_{p u r}-\imath g \vec{A}_{p u r} \times \vec{A}_{p u r}=0 \\
\overrightarrow{\mathcal{D}}_{p u r} \cdot \vec{A}_{f y s}=\vec{\nabla} \cdot \vec{A}_{f y s}-\imath g\left[A_{p u r}^{i}, A_{f y s}^{i}\right]=0
\end{array}
$$

Recall here that the color cross-product is not zero due to the matrix nature of $\vec{A}$. These constraints must now be solved perturbatively, but the effect is as one expects for a gauge transformation:

$$
\vec{A}_{f y s}^{\prime}=U \vec{A}_{f y s} U^{\dagger} ; \quad \vec{A}_{p u r}^{\prime}=U \vec{A}_{p u r} U^{\dagger}-\frac{\imath}{g} U \vec{\nabla} U^{\dagger}
$$

For completeness, I also list the explicit time derivative parts of the equations:

$$
\begin{aligned}
& \partial_{t} A_{f y s}^{0}=\partial_{i} A^{0}+\partial_{t}\left(A^{i}-A_{f y s}^{i}\right)-\imath g\left[\left(A^{i}-A_{f y s}^{i}\right),\left(A^{0}-A_{f y s}^{0}\right)\right] \\
& \partial_{i} A_{\text {pur }}^{0}=-\partial_{t} A_{\text {pur }}^{i}+\imath g\left[A_{\text {pur }}^{i}, A_{\text {pur }}^{0}\right]
\end{aligned}
$$

\section{A. Alternative Proposals}

Jaffe and Manohar[8] solve the problem on the light-cone, where there is only helicity to deal with. As cited above, Ji[7] ignores the conflict with commutation relations in favor of classic gauge invariant operators, and Leader[9] takes the same viewpoint. Wakamatsu[10] proposes a different apportionment of our decomposition, which has two problems: It again violates the angular momentum commutation algebra and its frame independence actually 
conflicts with Lorentz invariance, as it is only $J$, the total angular momentum, that is covariant - as we showed above, boosts reduce rest frame spin, shifting it to orbital angular

momentum. Finally, Cho et al.[11] propose a decomposition between gluons that they identify as "valence" or as "binding" gluons. This also appears to violate the angular momentum commutation algebra.

\section{CONCLUSION}

The physical component of a vector gauge field can be identified in a gauge covariant fashion. The gauge covariant derivatives needed to extract orbital angular momentum (and mechanical momentum) of fermions coupled to the gauge field must include only the nonphysical, pure gauge part of the vector gauge field so that: Both gauge invariance and canonical commutation relations are satisfied in order to allow physical interpretation of the matrix elements of these operators.

This work was carried out in part under the auspices of the National Nuclear Security Administration of the U.S. Department of Energy at Los Alamos National Laboratory under Contract No. DE-AC52-06NA25396. I thank the organizers of the Cairns Pacific Spin 2011 meeting for arranging it in this lovely location and for inviting me to speak.

[1] A. Airapetian et al. (HERMES Collaboration), Phys. Rev. D 75, 012007 (2007); V. Y. Alexakhin et al. (COMPASS Collaboration), Phys. Lett. B 647, 8 (2007).

[2] Xiang-Song Chen, Xiao-Fu Lü, Wei-Min Sun, Fan Wang and T. Goldman, Phys. Rev. Lett. 100, $232002(2008)$.

[3] D. V. Ahluwalia, T. Goldman and M. B. Johnson, Mod. Phys. Lett. A 9, 439 (1994). hep-th/9307118

[4] T. Goldman, Phys. Rev. D 15, 1063 (1977).

[5] E. B. Manoukian, J. Phys. G: Nucl.Phys. 13, 1013 (1987).

[6] D. Singleton and V. Dzhunushaliev, Found. Phys. 30, 1093 (2000).

[7] X. Ji, Phys. Rev. Lett. 78, 610 (1997).

[8] R. L. Jaffe and A. Manohar, Nucl. Phys. B 337, 509 (1990). 
[9] Elliot Leader Phys.Rev.D 83, 096012 (2011).

[10] M. Wakamatsu, Phys. Rev. D 81, 114010 (2010).

[11] Y. M. Cho, Mo-Lin Ge, D. G. Pak, and Pengming Zhang, arXiv:1102.1130 [nucl-th] (2011). 